\title{
Mitochondria: transportation, distribution and function during spermiogenesis*
}

\author{
Xiao Sun, Wan-Xi Yang
}

The Sperm Laboratory, Institute of Cell Biology and Genetics, College of Life Sciences, Zhejiang University, Hangzhou, China. Email: wxyang@spermlab.org

Received 19 March 2010; revised 29 March 2010; accepted 30 March 2010.

\begin{abstract}
Spermiogenesis is a dynamic process which includes organelle reorganization and new structure formation. The morphology and distribution of the mitochondria in germ cells change to accommodate the cellular requirement. Multiple molecular motors and related proteins participate in carrying and anchoring mitochondria to the midpiece during spermiogenesis and this process is regulated precisely. Energetic metabolism provides energy for cellular activity and influences sperm survival and motility directly. Ubiquitination of mitochondria takes place during spermiogenesis, which has been implicated in sperm quality control and mitochondrial inheritance. In light of the essential roles of mitochondria in energy production, calcium homeostasis and apoptosis, mitochondria dysfunction cause severe human diseases, such as male infertility. The present study paves a way for a more detailed exploration of the biology of mitochondria during spermiogenesis.
\end{abstract}

Keywords: Spermiogenesis; Mitochondria; Molecular Motors; Sperm Motility; Ubiquitination; Infertility

\section{INTRODUCTION}

Spermiogenesis is the last phase of spermatogenesis, where spherical spermatids differentiate into elongated spermatozoa in mammals [1,2]. Dynamic and pronounced changes occur in the process of spermiogenesis, including the formation of a condensed and elongated nucleus in the head, a well shaped acrosome flattened along the large portion of the nucleus, as well as a long flagellum. The flagellum is composed of cytoskeletal components and mitochondria provide the motors for sperm motility.

Mitochondria have a significant role in energy production, calcium homeostasis and apoptosis. Both sperm

\footnotetext{
*Grant sponsor: National Natural Science Foundation of China, Grant number: No. 30671606 and 40776079; National Basic Research Program of China (973 Program), Grant number: No 2007CB948104.
}

biosynthesis and motility require ATP. Recent findings indicate that two metabolic pathways are providing ATP for normal sperm function: oxidative phosphorylation in the midpiece and glycolysis in the principal piece [3-7]. Mitochondria are dynamic both in their morphological transitions and distribution. Several kinds of molecular motors have been indentified to be responsible for transporting mitochondria, including microtubule-dependent kinesins and dyneins, and microfilament-dependent myosins [8-14]. Several related proteins also function in transporting and docking of mitochondria [15]. Mitochondria provide maternal inheritance, and ubiquitination of mitochondria takes place to facilitate their degradation after fertilization [15]. Due to the indispensable role of mitochondria during fertilization, defect of sperm mitochondria or associated proteins can lead to male infertility.

In this review we mainly focus on the morphology and distribution of mitochondria, as well as the functions of this organelle during spermiogenesis. Furthermore, based on the transporting mechanisms within somatic cells, we attempt to define how mitochondria migrate to the midpiece, and to describe some related proteins that have been revealed in the mitochondria anchoring in the midpiece. Then, we review briefly about two energetic metabolisms that provide ATP for sperm motility, the ubiquitin modification during spermiogenesis, and finally talk about some mitochondria-related male infertility.

\section{DISTRIBUTION AND MORPHOLOGICAL CHANGES OF MITOCHONDRIA DURING SPERMIOGENESIS}

Spermiogenesis is a complicated morphogenesis occurring in the seminiferous epithelium of the testis during which the haploid spermatid is transformed into the finely shaped spermatozoon. Spermiogenesis has been divided into four phases in mammals: Golgi phase, cap phase, acrosome phase and maturation phase [1,16-19]. 
The different stages show their own features. At the early stages of spermiogenesis (Golgi and acrosome phase), the spermatids are round; vesicles originated from the Golgi apparatus migrate towards the pole where the pre-acrosomal vesicle locates; the axoneme emerges from the distal centriole and grows actively. At the acrosome phase, the nucleus initiates to elongate and condense, the mitochondria begin to migrate to the opposite pole of the pre-acrosome. During the maturation phase, the ODFs (outer dense fibers) grow rapidly outside of the axoneme, the mitochondria begin to elongate and condense to form the mitochondrial sheath; at the same time, the acrosome is well developed, the spermatids discard most of the cytoplasm defined as the residual body to become mature spermatozoa.

The flagellum of the mammalian spermatozoon consists of four distinct regions: the connecting piece, the mid piece, the principal piece, and the short end piece. In the central part of the mid piece, the axoneme is composed of a " $9+2$ " array of microtubules, nine ODFs surround the axoneme, and mitochondria are positioned in four helices along the ODFs [19-21]. The axoneme and seven ODFs surrounded by the fibrous sheath designate as the principal piece, while the end piece only includes the axoneme. During spermiogenesis, the mitochondria undergo dramatic changes both in morphology and location. In rat, the normal mitochondria with lamellar cristae are oval in shape. We observed the main events that happened during spermiogenesis in rat which supported the conclusion by De Martino et al. (1979) [22]. Our data show that, during the Golgi phase, cap phase and the early acrosome phase of spermiogenesis, the small, round or oval condensed mitochondria are distributed at the cell periphery, with its inner space flattened (Figures 1 and 2). The number of the mitochondria increases considerably. Therefore, the mitochondria are efficient to produce sufficient energy for exchanging metabolites between the adjacent Sertoli cells and spermatids or between the nucleus and the cytoplasm. The mitochondria exhibit convoluted cristae at the late acrosome phase and early maturation phase. We found that part of them move to the flagellum, whereas the remaining start to aggregate (Figure 3). These aggregated mitochondria are discarded within the residual bodies which are disposed by phagocytosis through the Sertoli cells or by autolysis in the mature spermatids [23]. Our data show that, at the late maturation phase, the mitochondria begin to condense and to elongate, and finally show a crescent shape along the ODFs in the mid piece (Figures 4 and 5). Mitochondria are supposed to generate ATP efficiently and rapidly. The length of the midpiece is similar in the same species, whereas it is obviously different among different species [24]. Possibly

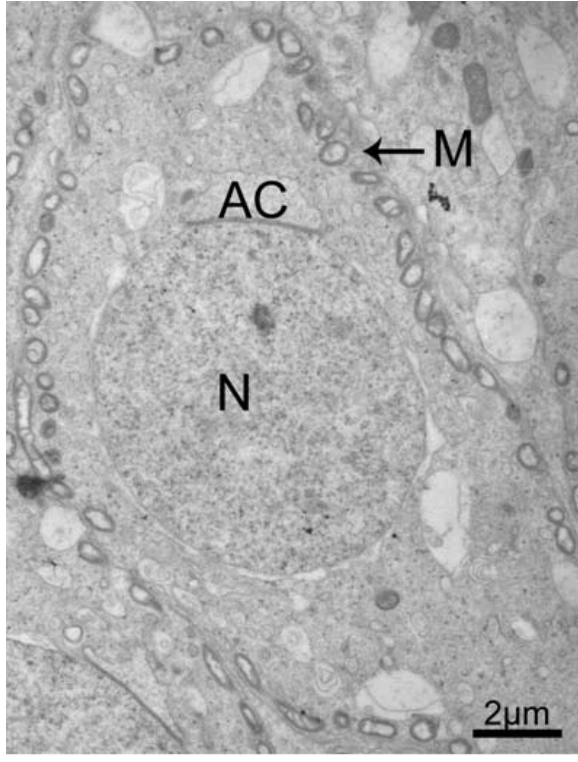

Figure 1. Mitochondria distribute at the cell periphery at the cap phase during rat spermiogenesis (black arrows in 1). $\mathrm{M}$ mitochondria, $\mathrm{AC}$ acrosome, $\mathrm{N}$ nucleus.

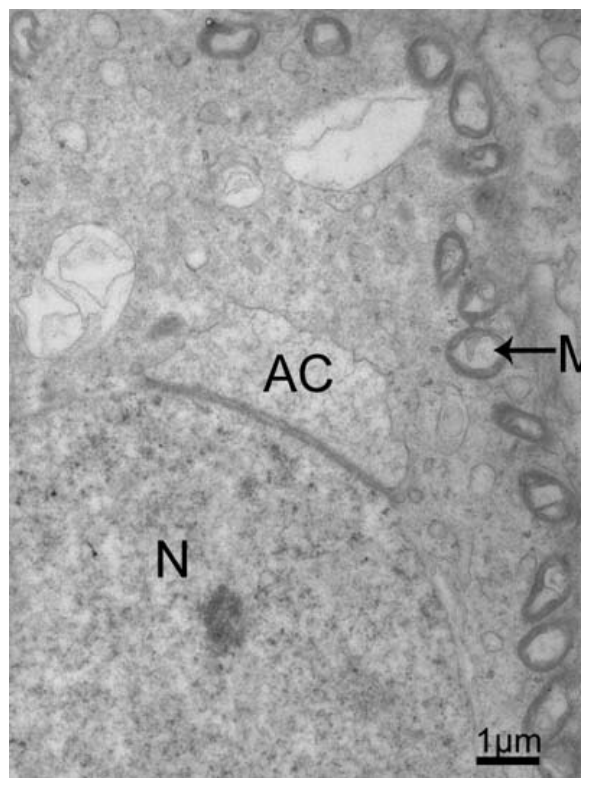

Figure 2. The magnification of partial Figure 1.

the whole length of the midpiece is responsible for energy supply when the fertilization process begins. A recent work by Ho et al. (2007) proposed a new three dimensional model to illustrate the mitochondrial arrangement in the mid piece in mouse. Initially, four dextral mitochondrial helices surround the outer dense fibers at stage 1. Subsequently, two mitochondria of the opposing arrays form circular structures at stage 2 . Then, staggered mitochondria form the sinistral double helix at 


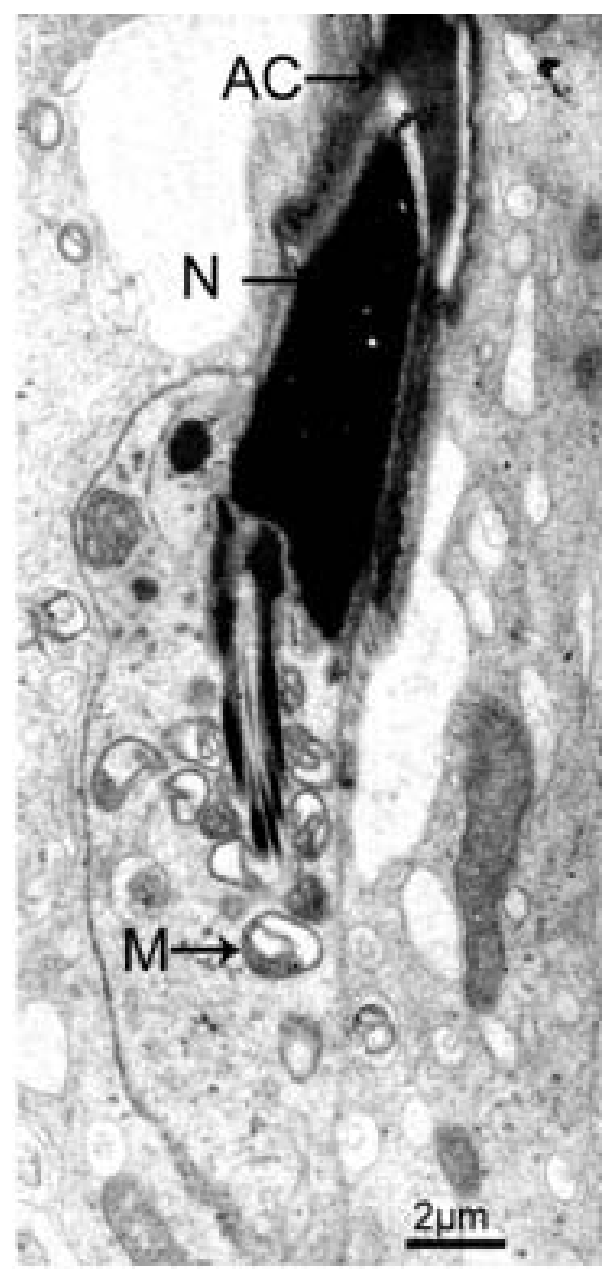

Figure 3. Part of mitochondria has been transported to the midpiece at the maturation phase.

stage 3. At the last stage, the crescent-shaped mitochondria transform to a rod-shaped form [25]. These observations provoke the question how the number, morphology and distribution of the mitochondria are controlled during spermatid differentiation.

Ultrastructure of the spermatozoa displays special features to adapt to the particular environment where fertilization takes place. In marine and freshwater animals, the spermatozoa morphology is different from mammals, exemplified by mollusk and fish.

Most of the marine bivalve mollusk spermatozoa are aquasperm, with a slightly curved or round or shortrodnucleus, a kind of species special acrosome (usually of conical shape), the midpiece consisting of one to six spherical mitochondria (four to five generally, such as Scapharca broughtoni, Figures 6 and 7) arranged in a circle that are surrounding a pair of centriole or a single large mitochondrion derived from fusion of several mitochondria, and a classical "9 + 2" flagellum [24,26-31]. Some cephalopod spermatozoa have a characteristic

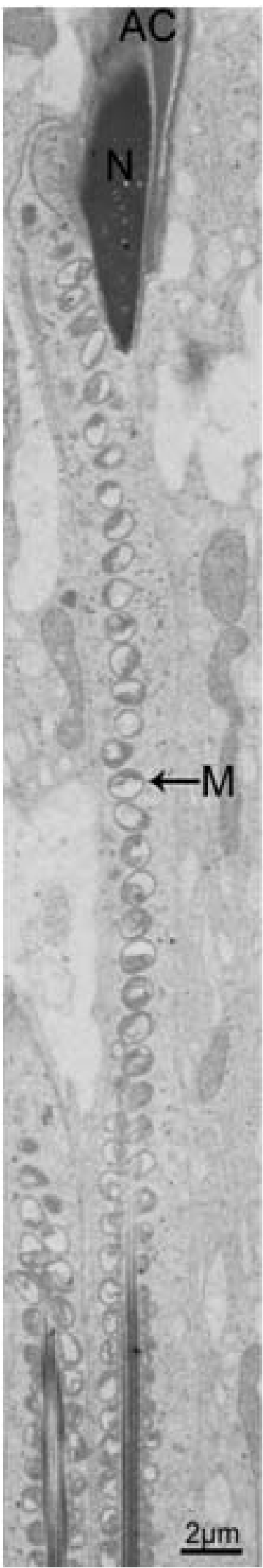

Figure 4. The mature spermatozoon of rat through head and midpiece. 


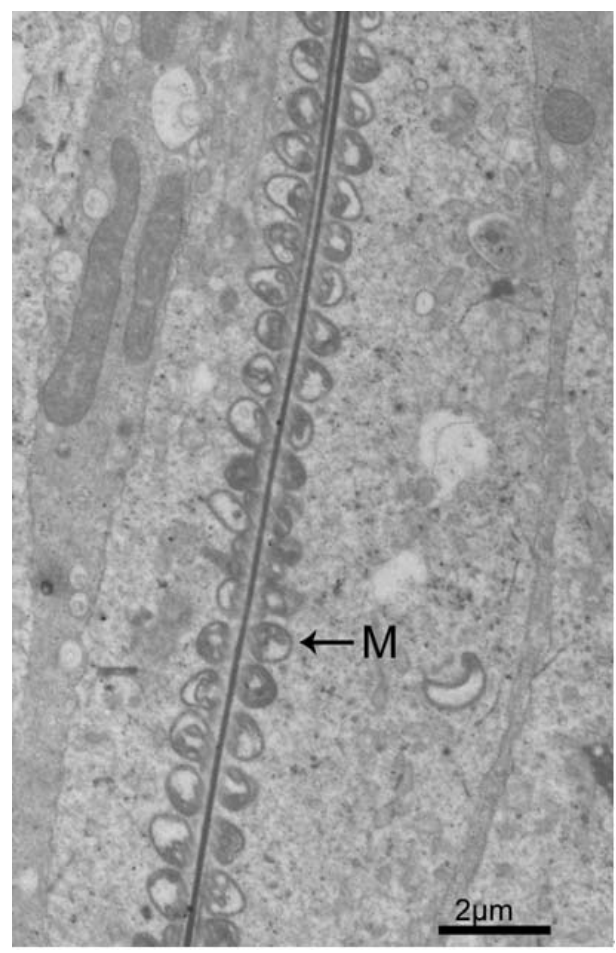

Figure 5. Mitochondrial localization in midpiece.

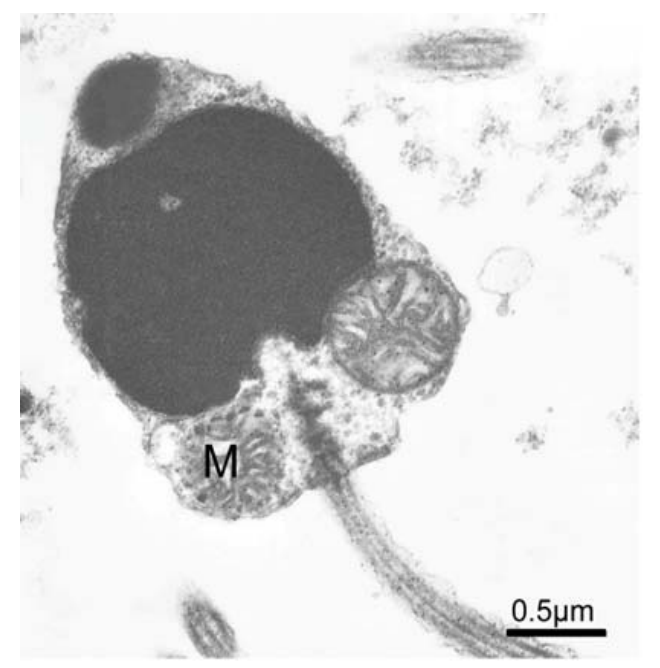

Figure 6. Typical spermatozoon of Scapharca broughtoni, two mitochondria visible.

acrosome with periodic and conical bands, and the mitochondrial number ranges from 9 to 11 in the midpiece, exemplified by Octopus tankahkeei [32-35] (Figures 8 and 9). Some kinds of teleostean spermatozoa belong to the aquasperm. Taking bony fish of the Perciformes as an example, their spermatozoa have a round head with a spherical nucleus, showing no acrosome, but a short midpiece with a cytoplasmic channel, and a flagellumwith typical axonemes [29,36,37]. Gusmão-Pompiani et al. (2005) found that in the marine teleost family Sciae-

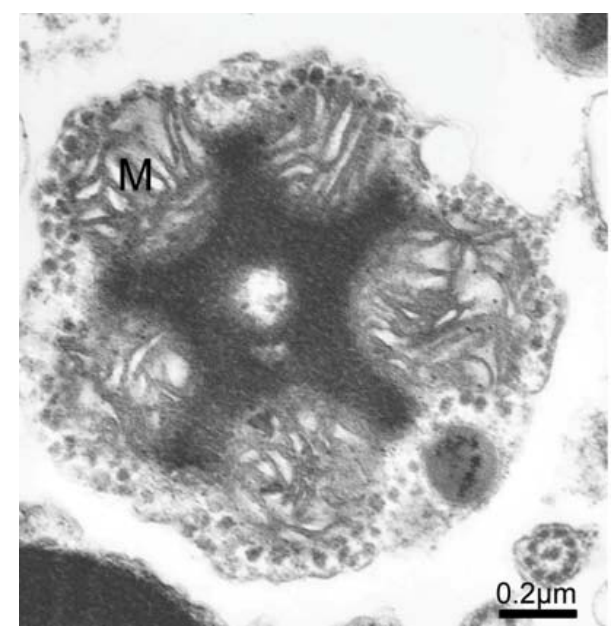

Figure 7. Cross section of midpiece in spermatozoon of Scapharca broughtoni, five mitochondria visible.

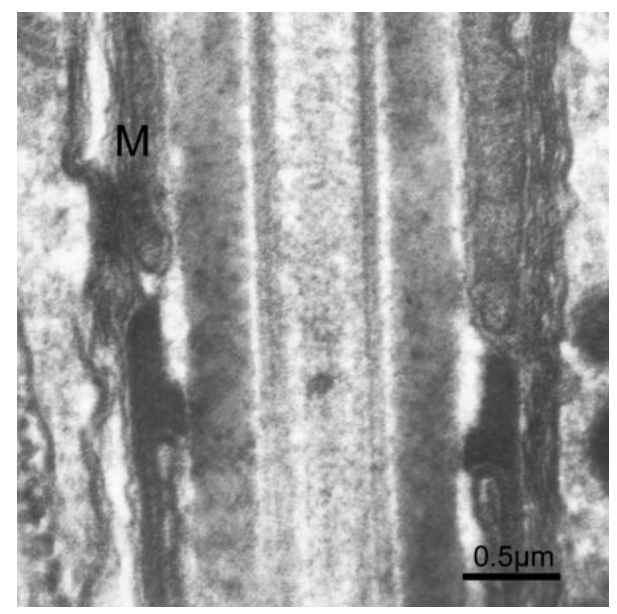

Figure 8. Joint between the midpiece and principal piece of the spermatozoon in Octopus tankahkeei.

nidae, there are less than ten spherical or elongate mitochondria which are located close to the nucleus and are arranged in one or two layers at the basal part of the mid piece [37]. Some exceptions exist in the Polynemidae, which are characterized by an arched nucleus surrounded by a large ring of mitochondria with a "C" shape in cross section [37]. The number of mitochondria in the midpiece in mollusks and teleostei is less prominent than in mammals.

Gage (1998) demonstrated that the flagellum length and the head length show a positive association with the mid piece length, these components may have co-evolved to provide effective fertilization [38]. The sperm swimming speed shows a positive ratio with sperm length, males with high sperm motility have higher fertilization success in mammals [39]. Mollusk and teleost employ external or internal fertilization. Sperm competition in the 


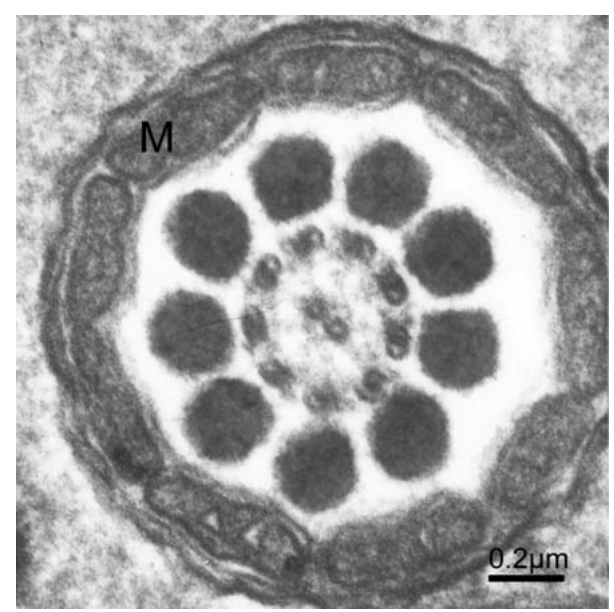

Figure 9. Cross section of the spermatozoon at the midpiece of the tail, showing 9 mitochondria.

course of external fertilization is mainly provided through the sperm number. Sperm longevity is negatively related to sperm length. Mean sperm length is shorter in free spawners compared to teleost with internal fertilization [40].

Sperm ultrastructure also reflects an adaption of the fertilization pattern to the environment. The flagellum becomes a longer mid piece containing a higher number of mitochondria and a fibrous sheath as an adaptation to internal fertilization. Such structural differences will enhance sperm motility which is necessary for successful fertilization.

\section{THE TRANSPORT OF MITOCHONDRIA}

The interaction between mitochondria and the cytoskeleton is essential for mitochondrial movement within cells. It was demonstrated that mitochondria move along the cytoskeleton depending on various molecular motors [12]. Different motors associate with mitochondria by docking intermediates such as adaptor proteins. Microtubule-dependent motors and actindependent motors are responsible for mitochondrial movement and docking. The head domain of two kinds of motors consists of a track binding site and an ATP catalytic site, on the other hand, the tail domain is responsible for recognizing and binding to diverse cargoes.

The microtubules show polarity within cells, with the minus end pointing to the centrosome and the plus end pointing to the cell periphery. Mitochondria move along the microtubule in a bidirectional manner, they usually stop and change directions under regulation to satisfy the cell metabolism. Kinesins and dyneins are microtubule dependent motors. The kinesin superfamily includes three classes of kinesins: N-terminus (KIN-N), C-terminus (KIN-C) and M-terminus (KIN-M). KIN-N moves to- ward the plus end of the microtubules and is responsible for anterograde transport in axons, whereas the cytoplasmic dyneins and KIN-C move to the minus end and are in charge of retrograde transport [41,42]. KIF1B and KIF5B belonging to KIN-N have been proposed to be involved in mitochondrial anterograde transport in axons [11]. KIF1B belonging to the kinesin 3 family is a monomeric motor participating in mitochondrial transport [10]. KBP (KIF1 binding protein) can regulate mitochondrial movement by increasing the motility of $\mathrm{KIF} 1 \mathrm{~B} \alpha$ by a mechanism that remains unknown as yet, which is clear that it does not serve as an adaptor [43]. Mitochondria aggregate around the nucleus when kif5B (a member of kinesin-1) is knocked out, which implicates that kif5B takes part in anterograde transport [9]. Various adaptors have been indentified in coupling kinesins to mitochondria, including the kinesin light chain (KLC), miro and milton complex, syntabulin, APLIP1, Miro and Grif-1 complex [44-48]. Different KLC isoforms target kinesins to different cargoes, and one type of KLC functions as an adaptor between mitochondria and kinesin-1 [44]. However, it was recently suggested that KLC is not necessary for kinesin-1 to transport mitochondria when miro-milton complexes are present in cultured cells [47]. It is therefore possible that $\mathrm{KLC}$ and miro-milton function together to transport mitochondria, each can substitute another when it is defective in a functional compensation mechanism. Miro anchors the mitochondria outer membrane by its tail domain, recruiting kinesin to form a functional complex in an indirect or direct manner. Glater et al. (2006) found that miro and milton form a complex linking mitochondria to kineisn-1 in Drosophila. In addition, milton also has a role in regulating the transport through diverse splicing patterns, by providing various modifications or by changing the state of miro [47]. In mammalian neurons, miro associates with Grif-1 to mediate mitochondrial motility in a signaling dependent way [48]. There are other findings in support of this. Miro contains GTP and $\mathrm{Ca}^{2+}$ binding domains and it functions as a linker. Miro also serves as a signal integrator and $\mathrm{Ca}^{2+}$ sensor, which has a role in regulating mitochondrial shape and transportation through interaction with cell signal transduction [49-51]. On the other hand, miro can bind kinesin independently [50]. Moreover, miro can prolong the process of kinesin and dynein on microtubules, by which it facilitates the mitochondria anterograde and retrograde transport [51]. Syntabulin, a membrane associated protein, acts as a kinesin-1 adaptor involved in mitochondria transport in mammalian neurons [45]. Whether syntabulin targets to mitochondria membranes directly or through other proteins requires further research. APLIP1, a kind of c-Jun $\mathrm{N}$-terminal kinase (JNK) interacting protein (JIP1), has been demonstrated to affect both kinesin and dynein based mitochondria transport, maybe acting as both kinesin and 
dynein linkers [46]. JNK signaling pathway possibly influences mitochondria transport by dissociating kinesin and APLIP1 or other related proteins [52].

Whether these different kinds of kinesin-adaptors work collectively to transport mitochondria, or in an individual manner by responding to different signaling pathways remain to be studied. The same holds for the molecular mechanisms that regulate motors to perform their action in the right sequence and the right place.

The main molecular motor responsible for minus-end transport along the microtubule is cytoplasmic dynein. It is involved in retrograde mitochondrial movement in axons. Dynein and dynactin form a complex to facilitate mitochondria transport [8]. In addition to control the distribution of mitochondria, dynein/dynactin complex also influence the Drp 1 recruitment to mitochondria. It takes part in the maintenance of normal mitochondrial morphology [53]. When Arp1 (a subunit of dynactin) is silent in Drosophila axons, the level of the kinesin heavy chain associating with the mitochondria is reduced which impair the anterograde and retrograde movement severely [54]. Conspicuously, similar to miro, dynactin has a role in coordinating bi-directional transport. However, the mechanism of dynactin function is not well understood. More interestingly, the retrograde movement of mitochondria is limited with kinesin-1 inhibition. Further experiments demonstrate that kinesin-1, dynein and dynactin form a complex to associate with the mitochondria [55]. There is also evidence that KIF5 interacts with dynein directly and specifically with KLCs. It also interacts with the dynein intermediate chain [56]. Based on these observations, it is speculated that KIF5 and dynein may serve as each other's cargo. Another possibility is that kinesin and dynein may form a complex to coordinate the bi-directional transport. It is still unknown whether they function independently to facilitate the transport in two opposite direction, or there exist other mechanisms to regulate the transport along the microtubules.

The activity of many proteins can be regulated by phosphorylation and dephosphorylation through protein kinases. Some signal pathways have been involved in mitochondria movement. Kinesins can bind to cargoes through kinesin light chains (KLC). KLC are hyperphosphorylated in the TNF signal pathway, then the phosphorylated KLC represses the activation of kinesin and leads to clusters of mitochondria around the nucleus [57]. NGF and PI3-kinase pathways also influence the mitochondrial distribution. NGF serves as a stop signal in neurons [58]. When the axons are stimulated by NGF, only the mitochondria begin to aggregate at the stimulated place [58]. Further experiments demonstrated that this process is regulated through PI 3-kinase by affecting the membrane receptors, a NGF downstream pathway
[59]. The JNK pathway has been demonstrated to influence mitochondrial transport by segregating the linkers and the cargoes [46,52].

However, whether different kinesin motors are walking in the same direction and binding to the cargoes simultaneously? What is the molecular mechanism to regulate the motors activities preciously? All these remain to be studied further.

While cargoes make long range transport on microtubules, actin filaments and myosins are responsible for short range transport within cells. Myosins are actin-based molecular motors, which are fundamental in cellular transport. Their motor domain is responsible for actin binding, and the tail domain for cargo binding. In budding yeast, myo2, a member of the myosin $\mathrm{V}$ family, plays a major role in mitochondrial motility and morphology, the transporting and docking of mitochondria on the actin filaments without myo2 are abolished [13]. Ypt11p, a small rab GTPase, may serve as an adaptor for myo2 to bind to mitochondrial membranes [60]. Recently, a novel class myosin, myo19 has been proposed to associate with mitochondria in human [14]. It is the first myosin discovered to be involved in the transportation of mitochondria in vertebrate cells. Further characterization of more myosin candidates and linking proteins may help us to understand mitochondria transport on the microfilaments. Considering all these results, it is reasonable to assume that molecular motors work together to dispatch the mitochondria in a way that their function can optimally be performed. We propose a model for mitochondria transport in somatic cells based on the available evidence (Figure 10). Several aspects need to get refined. These include the interaction and regulatory mechanism among multiple adaptors, molecular motors and the mitochondria. How various motors coordinate to facilitate the transport, and how the motors are transported to the location where they become functional remain unknown.

\section{TRANSPORT AND ANCHORING OF MITOCHONDRIA DURING SPERMIOGENESIS}

Similar to somatic cells, mitochondria transport during spermiogenesis has not been investigated in detail. How the mitochondria are delivered to the developing sperm tail is not clear as yet. Intramanchette transport (IMT) and intraflagellar transport (IFT) are involved in the sperm head and tail development, both of which are related to molecular motors associating with cargoes that are walking along the cystoskeletal elements [61]. The manchette, a transient structure, appears when the nucleus begins to elongate and disappears when the spermatid is well formed. IMT consists of both microtubule based and F-actin based transport. Kinesin II, dynein, myosin 


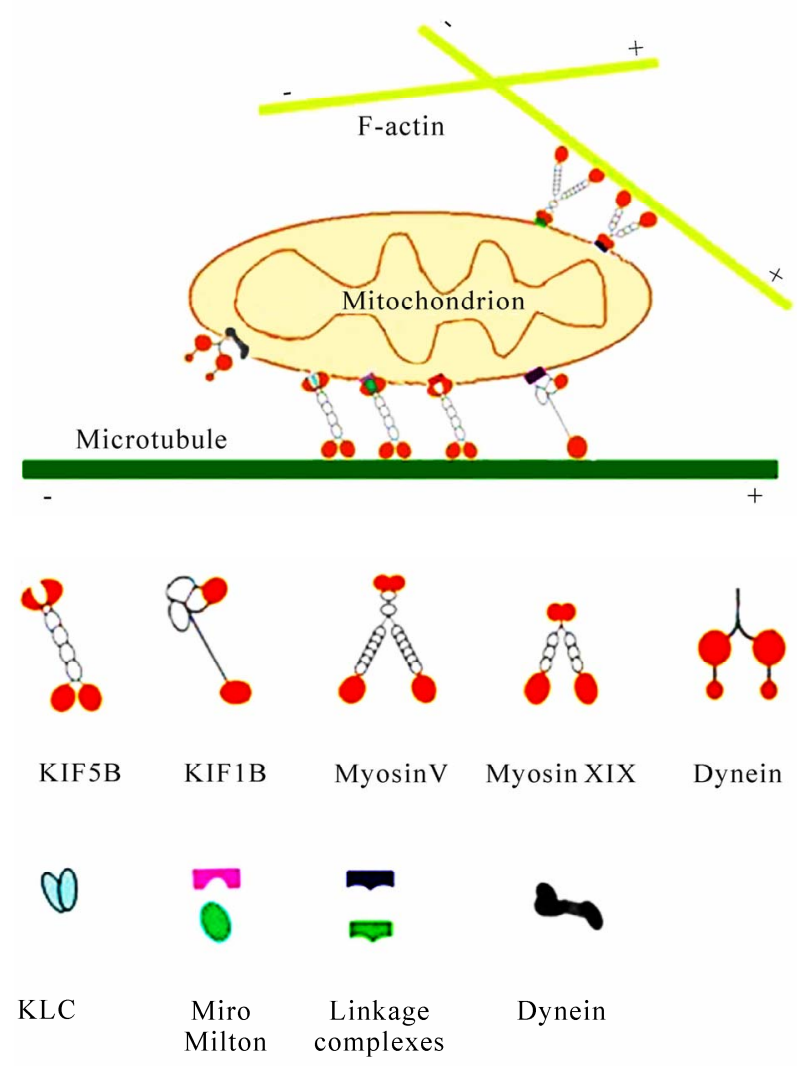

Figure 10. Diagrammatic representation of mitochondrial transport along the cystoskeletal elements in mammalian somatic cells. Motor proteins target mitochondria through special linkage complex. For instance, KIF5B associated mitochondria by KLC, Miro and Grif-1; Dynein and Dynactin form a complex to carry mitochondria. Myosin V and Myosin XIX transport mitochondria walk along the F-actin.

Va have been indentified to localize on the manchette, kinesin II is also involved in intraflagellar transport [62-66]. Further evidence supports that motors cooperate to facilitate intraflagellar transport in Caenorhabditis elegans $[67,68]$. IMT and IFT deliver materials for the tail development during spermiogenesis, thus, it is plausible to speculate that mitochondria migrate to the midpiece by IMT and IFT (Figure 11).

Some proteins take part in mediating mitochondria morphology and their localization has been uncovered. Nectin-2, Spergen-1 and KLC are three identified proteins that associate mitochondria to ODFs $[69,70]$. Nectin-2, a kind of cell adhesion molecule, localizes in the middle piece in mature spermatozoa. In nectin-2 depleted mice, the mitochondria exhibit abnormal distribution: some spermatozoa have disorganized mitochondrial sheaths, and some mitochondria dislocalize on the head [69]. Spergen-1 is only expressed in late spermiogenesis from the elongating spermatid to the mature spermatozoon. It is localized at the mitochondrial surface in the
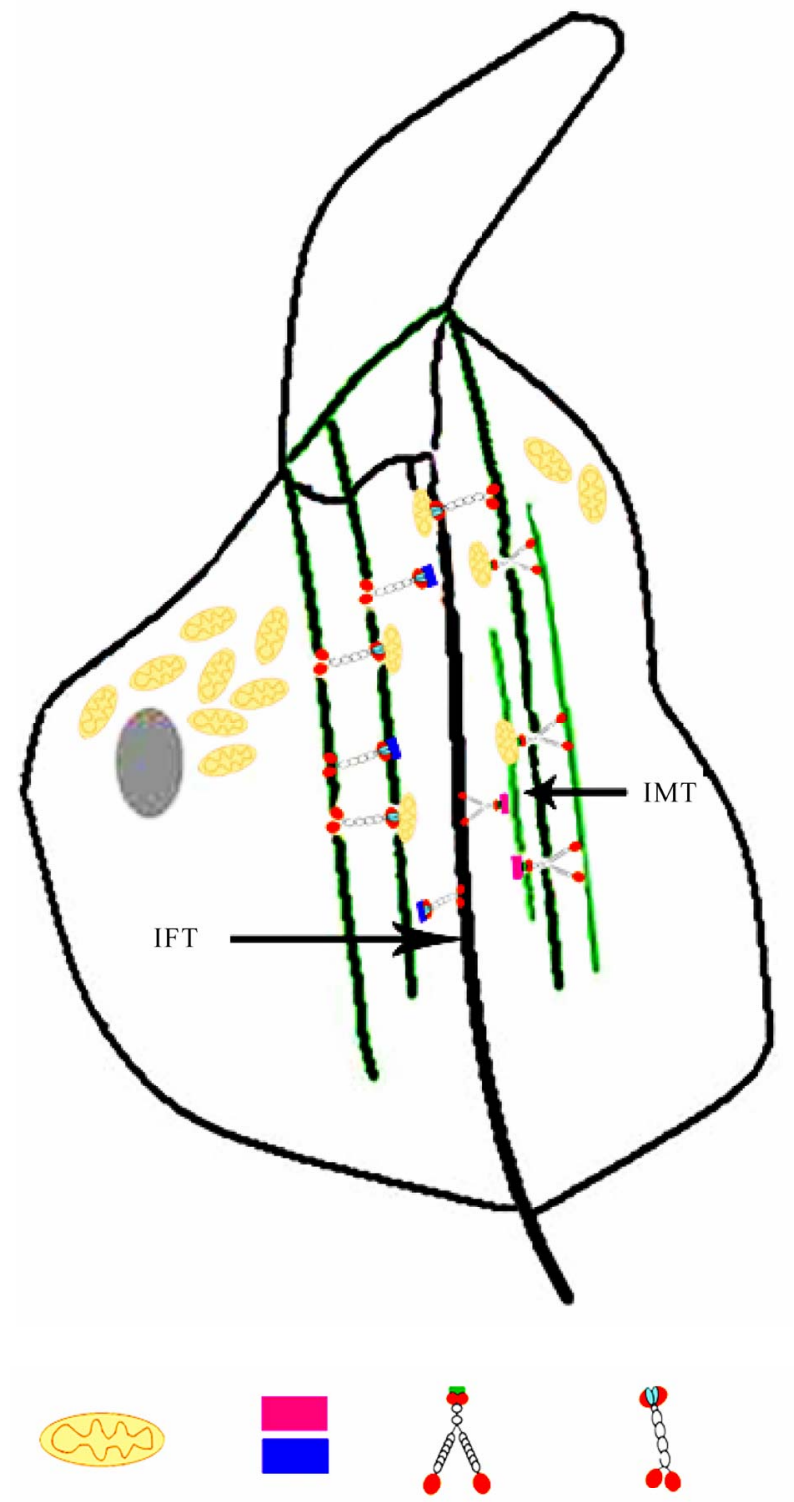

Mitochondrion

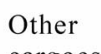

Myosin

and adaptors

Kinesin and adapyors

Figure 11. Diagrammatic representation about mitochondria transport at the early maturation phase in rat spermiogenesis. Kinesins and myosins work together to transport mitochondria and other cargoes to the tail through IMT and IFT.

mitochondria in cultured cells, it also contributes to couple mitochondria to ODFs during spermiogenesis [70-73]. Another spermatid specific protein is Kinesin Light Chain 3 (KLC3) and it has been found to connect to mitochondria through its TPR domain in the process of midpiece formation, more interestingly, KLC3 binds to ODFs using the HR domain in mature spermatozoa $[72,73]$. It is therefore possible that it links mitochondria to ODFs in a microtubule-independent way. Whether these linker proteins interact with each other or function 
alone at particular time remains to be determined. In GOPC or PICK1 knock out mice, the mitochondrial sheath arranges abnormally, which is the main feature of globozoospermia [74-76]. PICK1 locates at the mitochondrial membrane and recruits $\mathrm{PKC} \alpha$ implicated in phosphorylating some proteins [77]. Moreover, the PICK1/PKC $\alpha$ complex has been demonstrated to be involved in anti-apopotosis by affecting the Bcl2 factor [78]. In Pick $1^{-/-}$mice, the mitochondrial sheath is abnormal, and some mitochondria migrate to the deformed nucleus, resulting in low sperm motility [76]. However, the detailed mechanism of PICK1 involved in mitochondria sheath formation is unclear. Some observations revealed that the PKC $\alpha$ signaling pathway may modulate the activity of motors responsible for mitochondrial movement and participate in mitochondria mediated apoptosis of the abnormal sperm. Sertoli cells produce some paracrine mitochondrial maturation factor (PMMF) such as activin and follistatin, which regulate the shape and distribution of mitochondria during spermiogenesis in a paracrine way [79]. Accordingly, the mitochondrial membrane in the midpiece contains estrogen receptor $\beta$ and androgen receptors, indicating that hormones may influence the mitochondrial motility directly [80].

Further identification and characterization of related molecular motors and proteins can help us to understand more about the mechanism of spermiogenesis. Sperm cell culture and spermatogenic stem cell induction are the limiting factors, but these seem to be effective in investigating these issues.

\section{SPERM MOTILITY AND ENERGETICS}

Mammalian sperm need to sustain motility to reach and fertilize the egg. Mitochondrial aerobic respiration and glycolysis are two metabolic pathways to generate ATP for sperm movement and protein phosphorylation in sperm. Some evidence supports the notion that mitochondria are the only energy source for sperm motility. In mammal sperm, the overall mitochondrial volume shows a positive correlation with the flagellum length, hence, sperm motility is highly dependent on the number and size of mitochondria $[4,81]$. The sperm even keep their motility when the media contain no glucose or the metabolic pathway of glycolysis is inhibited [7]. The ATP being produced by mitochondria diffuse to the distal end to meet the high energy demand through shuttle mechanisms such as mediated by creatine kinase, adenylate kinase and phosphoglycerate kinase shuttles [5, 82]. However, different species may use different mechanism to deliver ATP produced by oxidative phosphorylation to the distal sperm tail.

Conversely, Malo et al. (2006) found that the longer the midpiece is, the slower the sperm moves; sperm motility also positively correlates with the head length and the length of the remaining tail [83]. Other mechanisms are possible to provide energy for sperm motility. Many observations reveal multiple glycolytic enzymes localized in the sperm fibrous sheath. Glycolysis contributes largely to sperm motility. In addition, glycolysis is sufficient to maintain sperm motility when oxidative phosphorylation is inhibited $[5,6]$. A newly developed technology combining laser and microscopic systems provides a useful tool to study the relation between sperm motility and energy metabolism [84]. With this tool, Nascimento et al. (2008) discovered that glycolysis provides more ATP molecules than oxidative phosphorylation to maintain sperm motility in mammals [85]. The $\mathrm{Ca}^{2+}$ pathway and cAMP/PKA pathway are two important ways of signal transduction involved in sperm motility control [86-88]. Some other proteins such as protease activated receptor 2 , the epidermal growth factor receptor, and pathways including the PI3-K cascade mediate sperm motility $[89,90]$.

Both mitochondrial respiration and glycolysis provide energy during spermiogenesis. Whether these two pathways are stage specific, or might compensation each other remains unclear. It also remains open for future studies to what extent the two pathways are required in different species.

\section{UBIQUITINATION OF SPERM MITOCHONDRIA}

Ubiquitination, a significant post-translated modification, has multiple biological effects, involved in protein degradation, endocytosis and signaling, activation of transcriptional factors, cell cycle, cell differentiation, immune responses and others [91-94]. In this process, ubiquitin or ubiquitin-like proteins target the substrate through different modifications, such as by different numbers of ubiquitin conjugated, and by various types of linkers among ubiquitins. For instance, monoubiquitin usually results in the target degradation by proteosome or lysosomes.

Mitochondria are ubiquitinated during spermiogenesis. The defective mitochondria will be degraded after ubiquination. In normal mitochondria in the midpiece, cross-linking disulfide bonds cover the ubiquitin tag so as not to be proteolyzed until fertilization, which appears to be a mechanism to maintain the mitochondrial maternal inheritance [15]. Prohibitin is a constitutive protein localized on the mitochondrial membrane in the midpiece, which serves as an ubiquitinated substrate and determines the mitochondrial destination [95]. Defective sperm exhibit high level ubiquitination of mitochondria, they are discarded during spermiogenesis or in the epididymis. Ubiquitination of sperm mitochondria is useful in indentifying sperm quality and act as a criterion for infertility diagnosis. Deep and extensive studies on sperm mitochondria ubiquitination process will provide us with a better understanding of the molecular mecha- 
nisms involved, as well as develop more feasible strategies and drugs in clinical trials.

\section{MITOCHONDRIA IN MALE INFERTILITY}

Defects in mitochondrial transport processes, ion channels and metabolic pathways cause various diseases, such as Parkinson's and Alzheimer's disease. Asthenozoospermia defined by low sperm motility and oligospermia characterized by reduced sperm number are two main causes for male infertility. The mitochondrial sheath dysfunction is the main cause for asthenozoospermia [96].

Mitochondria produce ATP for sperm survival and fertilization. Defective mitochondria cause low sperm motility [97]. Mitochondrial DNA mutations also affect sperm motility [98]. Both point mutation and fragment deletions may lead to abnormal mitochondrial structure and function, which in turn may cause male infertility [97-101]. Quantitative conventional PCR and mutant mice models are useful in the identification of genes related to male infertility $[75,102,103]$. Mitochondria are a major source of reactive oxygen species (ROS) generation, leading to apoptosis, and excessive ROS is a cause of male infertility. Antioxidant compounds are beneficial to improve fertility, and may serve as preventive drugs [104]. From this point of view, we may employ genetic therapy that combined with other therapeuticas to cure diseases at their place of origin in the future.

Some chemical compounds are toxic to mitochondria and interfere with male fertility. An environmental contaminant, TCCD, induces oxidative stress, with the consequence of reduced male fertility [105]. Parabens, a kind of food preservatives, may affect mitochondrial respiration and apoptosis that is mediated by mitochondria [106]. Toxicity to mitochondria in testes may serve as a criterion for the estimation of chemical side effects in the future.

\section{CONCLUSIONS AND PERSPECTIVE}

Mitochondria have effects on cell metabolism, cell signaling and apoptosis. Considering this, the intracellular localization of mitochondria is a critical factor. Under related cell signal transduction and regulatory mechanisms, some molecular motors transport the mitochondria from the cell periphery to the midpiece, and then form the mitochondrial sheath consisting of four mitochondria helices in mammals. Comparing numbers, mitochondria are much less in molluscs and teleosts than in mammals, which imply an adaption to the internal fertilization. Energy for sperm motility is provided by different mechanisms in the different parts of the spermatozoon. Whereas oxidative phosphorylation takes place within mitochondria that are located in the midpiece, glycolysis takes place along the principal piece.
Ubiquitination assumes the responsibility for sperm quality control and maternal inheritance. To a great extend is male infertility caused by defective sperm and low sperm motility.

Since mitochondria more and more turned out to be relevant for human health, research on mitochondria deserves increased attention. Dynamics of mitochondrial structure, motility and function during spermiogenesis have become an exciting field of research. What kinds of molecular motors are involved, how these are coordinated to transport mitochondria, how oxidative phosphorylation and glycolysis are regulated, and the underlying ubiquitination mechanisms requires to be clarified. The same holds for mitochondria related pathogenic mechanisms in human diseases. Investigating these issues provide an intriguing approach to future studies, particularly at the molecular level.

\section{ACKNOWLEDGEMENTS}

We are grateful to all members of the Sperm Laboratory at Zhejiang University. This work was supported by the National Natural Science Foundation of China, Grant number: No. 30671606 and 40776079; National Basic Research Program of China (973 Program), Grant number: No 2007CB948104.

\section{REFERENCES}

[1] Abou-Haila, A. and Tulsiani, D.R. (2000) Mammalian sperm acrosome: Formation, contents, and function. Archives of Biochemistry and Biophysics, 379(2), 173-182.

[2] Fawcett, D.W. (1975) The mammalian spermatozoon. Developmental Biology, 44(2), 394-436.

[3] Kamp, G., Büsselmann, G. and Lauterwein, J. (1996) Spermatozoa: Models for studying regulatory aspects of energy metabolism. Experientia, 52(2), 487-493.

[4] Ruiz-Pesini, E., Diez, C., Lapeña, A.C., Pérez-Martos, A., Montoya, J., Alvarez, E., Arenas, J. and López-Pérez, M.J. (1998) Correlation of sperm motility with mitochondrial enzymatic activities. Clinical Chemistry, 44(8), 1616-1620.

[5] Krisfalusi, M., Miki, K., Magyar, P.L. and O'Brien, D.A. (2006) Multiple glycolytic enzymes are tightly bound to the fibrous sheath of mouse spermatozoa. Biology of Reproduction, 75(2), 270-278.

[6] Molloy, P.P., Goodwin, N.B., Côté, I.M., Reynolds, J.D., Gage, M.J. Mukai, C. and Okuno, M. (2004) Glycolysis plays a major role for adenosine triphosphate supplementation in mouse sperm flagellar movement. Biology of Reproduction, 71(2), 540-547.

[7] Ford, W.C.L. (2006) Glycolysis and sperm motility: Does a spoonful of sugar help the flagellum go round? Human Reproduction Update, 12(3), 269-274.

[8] Waterman-Storer, C.M., Karki, S.B., Kuznetsov, S.A., Tabb, J.S., Weiss, D.G., Langford, G.M. and Holzbaur, E.L. (1997) The interaction between cytoplasmic dynein and dynactin is required for fast axonal transport. Proceedings of the National Academy of Sciences, 94(22), 12180-12185

[9] Tanaka, Y., Kanai, Y., Okada, Y., Nonaka, S., Takeda, S., 
Harada, A. and Hirokawa, N. (1998) Targeted disruption of mouse conventional kinesin heavy chain, kif5b, results in abnormal perinuclear clustering of mitochondria. Cell, 93(7), 1147-1158.

[10] Nangaku, M., Sato-Yoshitake, R., Okada, Y., Noda, Y., Takemura, R., Yamazaki, H. and Hirokawa, N. (1994) KIF1B, a novel microtubule plus end-directed monomeric motor protein for transport of mitochondria. Cell, 79(7), 1209-1220.

[11] Hirokawa, N. and Takemura, R. (2005) Molecular motors and mechanisms of directional transport in neurons. $\mathrm{Na}$ ture Reviews Neuroscience, 6(3), 201-214.

[12] Boldogh, I.R. and Pon, L.A. (2007) Mitochondria on the move. TRENDS in Cell Biology, 17(10), 502-510.

[13] Altmann, K., Frank, M., Neumann, D., Jakobs, S. and Westermann, B. (2008) The class V myosin motor protein, myo2, plays a major role in mitochondrial motility in Saccharomyces cerevisiae. The Journal of Cell Biology, 181(1), 119-130.

[14] Quintero, O.A., Divito, M.M., Adikes, R.C., Kortan, M.B., Case, L.B., Lier, A.J., Panaretos, N.S., Slater, S.Q., Rengarajan, M., Feliu, M., and Cheney, R.E. (2009) Human Myo19 is a novel myosin that associates with mitochondria. Current Biology, 19(23), 2008-2013.

[15] Sutovsky, P. (2003) Ubiquitin-dependent proteolysis in mammalian spermatogenesis, fertilization, and sperm quality control: Killing three birds with one stone. Microscopy Research and Technique, 61(1), 88-102.

[16] Clermont, Y. and Leblond, C.P. (1955) Spermiogenesis of man, monkey, ram and other mammals as shown by the "periodic acid-schiff technique". American Journal of Anatomy, 96(2), 229-253.

[17] Bedford, J.M. and Nicander, L. (1971) Ultrastructural changes in the acrosome and sperm membranes during maturation of spermatozoa in the testis and epididymis of the rabbit and monkey. Journal of Anatomy, 108(3), 527-543.

[18] Clermont, Y., Oko, R. and Hermo, L. (1991) Cell biology of mammalian spermiogenesis. In: Desjardins, C. and Ewing, L.L., Ed., Cell and Molecular Biology of the Testis, Oxford University Press, New York, 332-376.

[19] Breed, W.G. (2004) The spermatozoon of Eurasian murine rodents: Its morphological diversity and evolution. Journal of morphology, 261(1), 52-69.

[20] Afzelius, B. (1959) Electron microscopy of the sperm tail; results obtained with a new fixative. Journal of Biophysical and Biochemical Cytology, 5(2), 269-278.

[21] Eddy, E.M., Toshimori, K. andO'Brien, D.A. (2003) Fibrous sheath of mammalian spermatozoa. Microscopy Research and Technique, 61(1), 103-115.

[22] Breucker, H., Schäfer, E. and Holstein, A.F. (1985) Morphogenesis and fate of the residual body in human spermiogenesis. Cell and Tissue Research, 240(2), 303-309.

[23] Fawcett, D.W. (1970) A comparative view of sperm ultrastructure. Biology of Reproduction Supplement, 2(2), 90-127.

[24] De Martino, C., Floridi, A., Marcante, M.L., Malorni, W., Barcellona, P.S., Bellocci, M. and Silvestrini, B. (1979) Morphological, histochemical and biochemical studies on germ cell mitochondria of normal rats. Cell and Tissue Research, 196(1), 1-22.
[25] Ho, H.C. and Wey, S. (2007) Three dimensional rendering of the mitochondrial sheath morphogenesis during mouse spermiogenesis. Microscopy Research and Technique, 70(8), 719-723.

[26] Buckland-Nicks, J. and Scheltema, A. (1995) Was internal fertilization an innovation of early bilateral evidence from sperm structure of a mollusc. Proceedings of Biological Sciences, 261(1360), 11-18.

[27] Healy, J.M., Keys, J.L. and Daddow, L.Y.M. (2000) Comparative sperm ultrastructure in pteriomorphia bivalves with special reference to phylogenetic and taxonomic implications. In Harper, E., Taylor, J.D. and Crame, J.A., Ed., Evolutionary Biology of the Bivalvia, Geological Society, London, Special Publications, 177, 169-190.

[28] Gwo, J.C., Yang, W.T., Sheu, Y.T. and Cheng, H.Y. (2002) Spermatozoan morphology of four species of bivalve (Heterodonta, Veneridae) from Taiwan. Tissue and Cell, 34(1), 39-43.

[29] Gwo, J.C., Chiu, J.Y., Lin, C.Y., Su, Y. and Yu, S.L. (2005) Spermatozoan ultrastructure of four Sparidae fishes: Acanthopagrus berda, Acanthopagrus australis, Lagodon rhomboids and Archosargus probatocephus. Tissue and Cell, 37(2), 109-115.

[30] Zhu, J.Q., Dahms, H.U. and Yang, W.X. (2008) Ultrastructure of the mature spermatozoon of the bivalve Scapharca broughtoni. (Mollusca: Bivalvia: Arcidae). Micron, 39(8), 1205-1209.

[31] Zhu, J.Q. and Yang, W.X. (2009) Ultrastructure of the mature spermatozoon of the bivalve Estellarca olivacea (Mollusca: Bivalvia: Arcidae) and its phylogenetic implications. Acta Biologica Hungarica, 60(1), 27-34.

[32] Maxwell, W.L. (1975) Spermiogenesis of Eusepia officinalis (L.), Loligo forbesi (Steenstrup) and Alloteuthits subuclata (L.) (Cephalopoda, Decapoda). Proceedings of Biological Sciences, 191(1105), 527-535.

[33] Selmi, M.G. (1996) Spermatozoa of two Eledone species (Cephalopoda, Octopoda). Tissue and Cell, 28(5), 613620.

[34] Zhu, J.Q., Yang, W.X., You, Z.J. and Jiao, H.F. (2005) The ultrastructure of the spermatozoon of Octopus tankahkeei. Journal of Shellfish Research, 24(4), 12031207.

[35] Roura, A., Guerra, A., González, A.F. and Pascual, S. (2010) Sperm ultrastructure in Bathypolypus bairdii and B. sponsalis (Cephalopoda, Octopoda). Journal of Morphology, 271(2), 143-151.

[36] Quagio-Grassiotto, I., Antoneli, F.N. and Oliveira, C. (2003) Spermiogenesis and sperm ultrastructure in Cichla intermedia with some considerations about Labroidei spermatozoa (Teleostei, Perciformes, Cichlidae). Tissue and Cell, 35(6), 441-446.

[37] Gusmão-Pompiani, P., Oliveira, C. and Quagio-Grassiotto, I. (2005) Spermatozoa ultrastructure in Sciaenidae and Polynemidae (Teleostei: Perciformes) with some consideration on Percoidei spermatozoa ultrastructure. Tissue and Cell, 37(3), 177-191.

[38] Gage, M.J. (1998) Mammalian sperm morphometry. Proceedings of Biological Sciences/The Royal Society, 265(1391), 97-103.

[39] Gomendio, M. and Roldan, E.R. (2008) Implications of diversity in sperm size and function for sperm competi- 
tion and fertility. International Journal of Developmental Biology, 52(5-6), 439-447.

[40] Ball, M.A. and Parker, G.A. (1996) Sperm competition games: External fertilization and "adapative" infertility. Journal of Theoretical Biology, 180(2), 141-150.

[41] Hirokawa, N., Noda, Y. and Okada, Y. (1998) Kinesin and dynein superfamily proteins in organelle transport and cell division. Current Opinion in Cell Biology, 10(1), 60-73.

[42] Hirokawa, N. (1998) Kinesin and dynein superfamily proteins and the mechanism of organelle transport. Science, 279(5350), 519-526.

[43] Wozniak, M.J., Melzer, M., Dorner, C., Haring, H.U. and Lammers, R. (2005) The novel protein KBP regulates mitochondria localization by interaction with a kinesinlike protein. BMC Cell Biology, 6(1), 1-15.

[44] Khodjakov, A., Lizunova, E.M., Minin, A.A., Koonce, M.P. and Gyoeva, F.K. (1998) A specific light chain of kinesin associates with mitochondria in cultured cells. Molecular Biology of the Cell, 9(2), 333-343.

[45] Cai, Q., Gerwin, C. and Sheng, Z.H. (2005) Syntabulinmediated anterograde transport of mitochondria along neuronal processes. The Journal of Cell Biology, 170(6), 959-969.

[46] Horiuchi, D., Barkus, R.V., Pilling, A.D., Gassman, A. and Saxton, W.M. (2005) APLIP1, a kinesin binding JIP-1/JNK scaffold protein, influences the axonal transport of both vesicles and mitochondria in Drosophila. Current Biology, 15(23), 2137-2141.

[47] Glater, E.E., Megeath, L.J., Stowers, R.S. and Schwarz, T.L. (2006) Axonal transport of mitochondria requires milton to recruit kinesin heavy chain and is light chain independent. The Journal of Cell Biology, 173(4), 545557.

[48] MacAskill, A.F., Brickley, K., Stephenson, F.A. and Kittler, J.T. (2009) GTPase dependent recruitment of Grif-1 by Miro1 regulates mitochondrial trafficking in hippocampal neurons. Molecular and Cellular Neuroscience, 40(3), 301-312.

[49] Frederick, R.L., McCaffery, J.M., Cunningham, K.W., Okamoto, K. and Shaw, J.M. (2004) Yeast Miro GTPase, Gem1p, regulates mitochondrial morphology via a novel pathway. The Journal of Cell Biology, 167(1), 87-98.

[50] MacAskill, A.F., Rinholm, J.E., Twelvetrees, A.E., Arancibia-Carcamo, I.L., Muir, J., Fransson, A., Aspenstrom, P., Attwell, D. and Kittler, J.T. (2009) Miro1 is a calcium sensor for glutamate receptor-dependent localization of mitochondria at synapses. Neuron, 61(4), 541-55.

[51] Russo, G.J., Louie, K., Wellington, A., Macleod, G.T., Hu, F., Panchumarthi, S. and Zinsmaier, K.E. (2009) Drosophila Miro is required for both anterograde and retrograde axonal mitochondrial transport. Journal of Neuroscience, 29(17), 5443-5455.

[52] Horiuchi, D., Collins, C.A., Bhat, P, Barkus, R.V., Diantonio, A. and Saxton, W.M. (2007) Control of a kinesincargo linkage mechanism by JNK pathway kinases. Current Biology, 17(15), 1313-1317.

[53] Varadi, A., Johnson-Cadwell, L.I., Cirulli, V., Yoon, Y., Allan, V.J. and Rutter, G.A. (2004) Cytoplasmic dynein regulates the subcellular distribution of mitochondria by controlling the recruitment of the fission factor dynaminrelated protein-1. Journal of Cell Science, 117(19),
4389-4400.

[54] Haghnia, M., Cavalli, V., Shah, S.B., Schimmelpfeng, K., Brusch, R., Yang, G., Herrera, C., Pilling, A. and Goldstein, L.S. (2007) Dynactin is required for coordinated bidirectional motility, but not for dynein membrane attachment. Molecular Biology of the Cell, 18(6), 20812089.

[55] Pilling, A.D., Horiuchi, D., Lively, C.M. and Saxton, W.M. (2006) Kinesin-1 and dynein are the primary motors for fast transport of mitochondria in drosophila motor axons. Molecular Biology of the Cell, 17(4), 20572068.

[56] Ligon, L.A., Tokito, M., Finklestein, J.M., Grossman, F.E. and Holzbaur, E.L. (2004) A direct interaction between cytoplasmic dynein and kinesin I may coordinate motor activity. Journal of Biological Chemistry, 279(18), 1920119208.

[57] De Vos, K., Severin, F., Van Herreweghe, F., Vancompernolle, K., Goossens, V., Hyman, A. and Grooten, J. (2000) Tumor necrosis factor induces hyperphosphorylation of kinesin light chain and inhibits kinesin-mediated transport of mitochondria. The Journal of Cell Biology, 149(6), 1207-1214.

[58] Reynolds, I.J. and Rintoul, G.L. (2004) Mitochondrial stop and go: Signals that regulate organelle movement. Science's STKE, 2004(251), 46.

[59] Chada, S.R. and Hollenbeck, P.J. (2003) Mitochondrial movement and positioning in axons: The role of growth factor signaling. The Journal of Experimental Biology, 206(12), 1985-1992.

[60] Itoh, T., Watabe, A., Toh-E, A. and Matsui, Y. (2002) Complex formation with Ypt11p, a rab-Type small GTPase, is essential to facilitate the function of myo2p, a class V Myosin, in mitochondrial Ddistribution in Saccharomyces cerevisiae. Molecular and Cellular Biology, 22(22), 7744-7757.

[61] Kierszenbaum, A.L. (2002). Intramanchette transport (IMT): Managing the making of the spermatid head, centrosome, and tail. Molecular Reproduction and Development, 63(1), 1-4

[62] Yoshida, T., Ioshii, S.O., Imanaka-Yoshida, K. and Izutsu, K. (1994) Association of cytoplasmic dynein with manchette microtubules and spermatid nuclear envelope during spermiogenesis in rats. Journal of Cell Science, 107(3), 625-633.

[63] Cole, D.G., Diener, D.R., Himelblau, A.L., Beech, P.L., Fuster, J.C. and Rosenbaum, J.L. (1998) Chlamydomonas kinesin-II-dependent intraflagellar transport (IFT): IFT particles contain proteins required for ciliary assembly in Caenorhabditis elegans sensory neurons. The Journal of Cell Biology, 141(4), 993-1008.

[64] Miller, M.G., Mulholland, D.J. and Vogl, A.W. (1999) Rat testis motor proteins associated with spermatid translocation (dynein) and spermatid flagella (kinesin-II). Biology of Reproduction, 60(4), 1047-1056.

[65] Kierszenbaum, A.L., Rivkin, .E and Tres, L.L. (2003) The actin-based motor myosin Va is a component of the acroplaxome, an acrosome-nuclear envelope junctional plate, and of manchette-associated vesicles. Cytogenetic and Genome Research, 103(3-4), 337-344.

[66] Hayasaka, S., Terada, Y., Suzuki, K., Murakawa, H., Tachibana, I., Sankai, T., Murakami, T., Yaegashi, N. and 
Okamura, K. (2008) Intramanchette transport during primate spermiogenesis: expression of dynein, myosin Va, motor recruiter myosin Va, VIIa-Rab27a/b interacting protein, and Rab27b in the manchette during human and monkey spermiogenesis. Asian Journal of Andrology, 10(4), 561-568.

[67] Ou, G., Blacque, O.E., Snow, J.J., Leroux, M.R. and Scholey, J.M. (2005) Functional coordination of intraflagellar transport motors. Nature, 436(7050), 583-587.

[68] Hancock, W.O. (2008) Intracellular transport: Kinesins working together. Current Biology, 18(16), R715-17.

[69] Bouchard, M.J., Dong, Y., McDermott, B.M. Jr., Lam, D.H., Brown, K.R., Shelanski, M., Bellvé, A.R. and Racaniello, V.R. (2000) Defects in nuclear and cytoskeletal morphology and mitochondrial localization in spermatozoa of mice lacking nectin-2, a component of cell-cell adherens junctions. Molecular and Cellular Biology, 20(8), 2865-2873.

[70] Doiguchi, M., Mori, T., Toshimori, K., Shibata, Y. and Iida, H. (2002) Spergen-1 might be an adhesive molecule associated with mitochondria in the middle piece of spermatozoa. Developmental Biology, 252(1), 127-137.

[71] Doiguchi, M., Yamashita, H., Ichinose, J., Mori, T., Shibata, Y. and Iida, H. (2002) Complementary DNA cloning and characterization of rat spergen-1, a spermatogenic cell-specific gene-1, containing mitochondriatargeting signal. Biology of Reproduction, 66(5), 14621470.

[72] Junco, A., Bhullar, B., Tarnasky, H.A. and van der Hoorn, F.A. (2001) Kinesin light-chain KLC3 expression in testis is restricted to spermatids. Biology of Reproduction, 64(5), 1320-1330.

[73] Zhang, Y., Oko, R. and van der Hoorn, F.A. (2004) Rat kinesin light chain 3 associates with spermatid mitochondria. Developmental Biology, 275(1), 23-33.

[74] Yao, R., Ito, C., Natsume, Y., Sugitani, Y., Yamanaka, H., Kuretake, S., Yanagida, K., Sato, A., Toshimori, K. and Noda, T. (2002) Lack of acrosome formation in mice lacking a Golgi protein, GOPC, 99(17), 11211-11216.

[75] Escalier, D. (2006) Knockout mouse models of sperm flagellum anomalies. Human Reproduction Update, 12(4), 449-461.

[76] Xiao, N., Kam, C., Shen, C., Jin, W., Wang, J., Lee, K.M., Jiang, L. and Xia, J. (2009) PICK1 deficiency causes male infertility in mice by disrupting acrosome formation. Journal of Clinical Investigation, 119(4), 802-812.

[77] Wang, W.L., Yeh, S.F., Chang, Y.I., Hsiao, S.F., Lian, W.N., Lin, C.H., Huang, C.Y. and Lin, W.J. (2003) PICK1, an anchoring protein that specifically targets protein Kinase $\mathrm{C} \alpha$ to mitochondria selectively upon serum stimulation in NIH $3 \mathrm{~T} 3$ cells. The Journal of Biological Chemistry, 278(39), 37705-37712.

[78] Wang, W.L., Yeh, S.F., Huang, E.Y.K., Lu, Y.L. and Wang, C.F. (2007) Mitochondrial anchoring of PKC $\alpha$ by PICK1 confers resistance to etoposide-induced apoptosis. Apoptosis, 12(10), 1857-1871.

[79] Meinhardt, A. and Wilhelm, B., Seitz, J. (1999) Expression of mitochondria marker proteins during spermatogenesis. Human Reproduction Update, 5(2), 108-119.

[80] Solakidi, S., Psarra, A.M., Nikolaropoulos, S. and Sekeris, C.E. (2005) Estrogen receptors $\alpha$ and $\beta$ (ER $\alpha$ and $\mathrm{ER} \beta$ ) and androgen receptor (AR) in human sperm: lo- calization of ER $\beta$ and AR in mitochondria of the midpiece. Human Reproduction, 20(12), 3481-3487.

[81] Cardullo, R.A. and Baltz, J.M. (1991) Metabolic regulation in mammalian sperm: mitochondrial volume determines sperm length and flagellar beat frequency. Cell Motility and the Cytoskeleton, 19(3), 180-188.

[82] Tombes, R.M. and Shapiro, B.M. (1985) Metabolite channeling: A phosphorylcreatine shuttle to mediate high energy phosphate transport between sperm mitochondrion and tail. Robert M. Cell, 41(1), 325-334.

[83] Malo, A.F., Gomendio, M., Garde, J., Lang-Lenton, B., Soler, A.J. and Roldan, E.R. (2006) Sperm design and sperm function. Biology Letters, 2(2), 246-249.

[84] Shi, L.Z., Nascimento, J.M., Chandsawangbhuwana, C., Botvinick, E.L. and Berns, M.W. (2008) An automatic system to study sperm motility and energetics. Biomedical Microdevices, 10(4), 573-583.

[85] Nascimento, J.M., Shi, L.Z., Tam, J., Chandsawangbhuwana, C., Durrant, B., Botvinick, E.L. and Berns, M.W. (2008) Comparison of glycolysis and oxidative phosphorylation as energy sources for mammalian sperm motility, using the combination of fluorescence imaging, laser tweezers, and real-time automated tracking and trapping. Journal of Cellular Physiology, 217(3), 745-751.

[86] Ho, H.C., Granish, K.A. and Suarez, S.S. (2002) Hyperactivated motility of bull sperm is triggered at the axoneme by $\mathrm{Ca}^{2+}$ and not cAMP. Developmental Biology, 250(1), 208-217.

[87] Turner, R.M. (2003) Tales from the tail: What do we really know about sperm motility? Journal of Andrology, 24(6), 790-803.

[88] Ishijima, S., Mohri, H., Overstreet, J.W. and Yudin, A.I. (2006) Hyperactivation of monkey spermatozoa is triggered by $\mathrm{Ca}^{2+}$ and Completed by cAMP. Molecular Reproduction and Development, 73(9), 1129-1139.

[89] Zitta, K., Albrecht, M., Weidinger, S., Mayerhofer, A. and Köhn, F. (2007) Protease activated receptor 2 and epidermal growth factor receptor are involved in the regulation of human sperm motility. Asian Journal of Andrology, 9(5), 690-696.

[90] Ashizawa, K., Omura, Y., Katayama, S., Tatemoto, H., Narumi, K. and Tsuzuki, Y. (2009) Intracellular signal transduction pathways in the regulation of fowl sperm motility: Evidence for the involvement of phosphatidylinositol 3-Kinase (PI3-K) cascade. Molecular Reproduction and Development, 76(7), 603-610.

[91] Hershko, A. and Ciechanover, A. (1998) The ubiquitin system. Annual Review of Biochemistry, 67, 425-479.

[92] Ciechanover, A., Orian, A. and Schwartz, A.L. (2000) Ubiquitin-mediated proteolysis: Biological regulation via destruction. Bioessays, 22(5), 442-451.

[93] Ciechanover, A. and Iwai, K. (2004) The ubiquitin system: From basic mechanisms to the patient bed. IUBMB Life, 56(5), 193-201.

[94] Mukhopadhyay, D. and Riezman, H. (2007) Proteosomeindependent functions of ubiquitin in endocytosis and signaling. Science, 315(5809), 201-205.

[95] Thompson, W.E., Ramalho-Santos, J. and Sutovsky, P. (2003) Ubiquitination of prohibitin in mammalian sperm mitochondria: Possible roles in the regulation of mitochondrial inheritance and sperm quality control. Biology of Reproduction, 69(1), 254-260. 
[96] Mundy, A.J., Ryder, T.A. and Edmonds, D.K. (1995) Asthenozoospermia and the human sperm mid-piece. Human Reproduction, 10(1), 116-119.

[97] Folgerq, T., Bertheussen, K., Lindal, S., Torbergsen, T. and Oian, P. (1993) Mitochondrial disease and reduced sperm motility. Human Reproduction, 8(11), 1863-1868.

[98] Spiropoulos, J., Turnbull, D.M. and Chinnery, P.F. (2002) Can mitochondrial DNA mutations cause sperm dysfunction? Molecular Human Reproduction, 8(8), 719-721.

[99] St John, J.C., Jokhi, R.P. and Barratt, C.L. (2005) The impact of mitochondrial genetics on male infertility. International Journal of Andrology, 28(2), 65-73.

[100] Shamsi, M.B., Kumar, R., Bhatt, A., Bamezai, R.N., Kumar, R., Gupta, N.P., Das, T.K. and Dada, R. (2008) Mitochondria DNA mutations in etiopathogenesis of male infertility. Indian Journal of Urology, 24(2), 150154.

[101] St John, J.C., Sakkas, D. and Barratt, C.L. (2000) A role for mitochondrial DNA and sperm survival. Journal of Andrology, 21(2), 189-199.

[102] Yan, W. (2009) Male infertility caused by spermiogenic defects: Lessons from gene knockouts. Molecular and Cellular Endocrinology, 306(1-2), 24-32.

[103] St John, J.C., Jokhi, R.P. and Barratt, C.L. (2001) Men with oligoasthenoteratozoospermia harbour higher numbers of multiple mitochondrial DNA deletions in their spermatozoa, but individual deletions are not indicative of overall aetiology. Molecular Human Reproduction, 7(1), 103-111.

[104] Lanzafame, F.M., La Vignera, S., Vicari, E. and Calogero, A.E. (2009) Oxidative stress and medical antioxidant treatment in male infertility. Reproductive Biomedicine Online, 19(5), 638-659.

[105] Latchoumycandane, C., Chitra, K.C. and Mathur, P.P. (2002). The effect of 2, 3, 7, 8-tetrachlorodibenzo-pdioxin on the antioxidant system in mitochondrial and microsomal fractions of rat testis. Toxicology, 171(2-3), 127-135.

[106] Tavares, R.S., Martins, F.C., Oliveira, P.J., RamalhoSantos, J. and Peixoto, F.P. (2008) Parabens in male infertility-Is there a mitochondrial connection? Reproductive Toxicology, 27(1), 1-7. 